\title{
A randomized trial of psychological intervention on perioperative anxiety and depression of patients with severe blepharoptosis undergoing autologous fascia lata frontal muscle suspension
}

\author{
Haiyan Cai, Suhui Xu \\ Ophthalmology Operating Room, Second Affiliated Hospital Zhejiang University College of Medicine, Hangzhou, China \\ Contributions: (I) Conception and design: H Cai; (II) Administrative support: S Xu; (III) Provision of study materials or patients: All authors; (IV) \\ Collection and assembly of data: All authors; (V) Data analysis and interpretation: All authors; (VI) Manuscript writing: All authors; (VII) Final \\ approval of manuscript: All authors. \\ Correspondence to: Suhui Xu. Ophthalmology Operating Room, Second Affiliated Hospital Zhejiang University College of Medicine, 88 Jiefang Road, \\ Shangcheng District, Hangzhou 310007, China. Email: 2195016@zju.edu.cn.
}

Background Perioperative negative emotion of surgical patients has a greater impact on surgical efficacy
and prognosis. The study aimed to analyze the effect of psychological intervention on perioperative anxiety
and depression of patients with severe blepharoptosis undergoing autologous fascia lata frontal muscle
suspension, and provide a reference for improving and optimizing patient care plans.

Methods: Ninety-two patients with severe blepharoptosis who underwent autologous fascia lata frontal muscle suspension in our hospital from February 2018 to January 2020 were selected as the research subjects. Patients were numbered according to the order of operation, and were divided into a control group (46 cases) and an observation group (46 cases) using a numerical random table method. Patients in both groups received routine nursing intervention during the perioperative period, and patients in the observation group also received psychological intervention during this period. The two groups of patients were evaluated using the Hamilton Anxiety Scale (HAM-A) and Hamilton Depression Scale (HAM-D). Numerical rating scale (NRS) results for surgical site pain were evaluated and compared between the groups. The perioperative compliance rate and nursing satisfaction rate of the two groups of patients were investigated and compared. Intraoperative and postoperative complications were collected, sorted, and compared between the two groups of patients.

Results: (I) The HAM-A and HAM-D scores of the observation group before and after surgery were significantly lower than those of the control group $(\mathrm{P}<0.05)$. (II) The NRS scores of patients in the observation group were markedly lower than those in the control group at $6 \mathrm{~h}$ and $24 \mathrm{~h}$ postoperatively $(\mathrm{P}<0.05)$. (III) The intra- and post-operative complication rate of the observation group was lower than that of the control group $(\mathrm{P}<0.05)$. (IV) The compliance rate and the total satisfaction rate of patients with the perioperative care in the observation group was considerably higher compared to that of patients in the control group $(\mathrm{P}<0.05)$.

Conclusions: Our results showed that psychological intervention can effectively alleviate the negative emotions in patients with severe blepharoptosis who underwent autologous fascia lata frontal muscle suspension, improve their compliance with medical care, reduce their pain, and increase their satisfaction rate. Therefore, psychological intervention has high clinical value.

Keywords: Severe blepharoptosis; autologous fascia lata frontal muscle suspension; Hamilton Anxiety; Hamilton Depression; compliance rate; pain numerical rating scale (pain NRS); complications; nursing satisfaction

Submitted Jan 27, 2021. Accepted for publication Mar 16, 2021.

doi: 10.21037/apm-21-345

View this article at: http://dx.doi.org/10.21037/apm-21-345 


\section{Introduction}

Eye surgery is currently the most prominent type of clinical cosmetic surgery, and the correction of ptosis is one of the most representative surgeries. The upper eyelid is composed of the levator facial muscle, the Whitnall ligament, the Müller muscle, blood vessels, and the lacrimal gland. Inflammation, trauma, tumors, and other secondary diseases of the body can cause partial or complete functional loss of the levator palpebral muscle and Muller smooth muscle, resulting in upper eyelid drooping (one or both upper eyelids lower than the normal position by more than $2 \mathrm{~mm}$ ) (1). There are also some patients with congenital ptosis. A levator muscle strength of less than $5 \mathrm{~mm}$ is clinically regarded as severe ptosis (2). In addition to covering the cornea, severe ptosis may also partially or completely cover the pupil, which has a serious impact on vision and visual function (3). Severe ptosis not only affects the patient's appearance, but also seriously affects their visual function, and simultaneously leads to the formation of facial defects. Clinical studies have shown that most patients with severe ptosis have varying degrees of inferiority complex and even psychological disorders, which seriously affects their physical and mental health (4).

Severe congenital ptosis is primarily treated with surgery; however, traumatic and secondary ptosis are first treated for the primary disease. However, if the patient's condition fails to improve after more than 6 months of systemic treatment, surgical treatment is required. The current surgical methods for ptosis include levator muscle shortening, frontal muscle flap suspension, etc. (5). Autologous fascia lata suspension is the most commonly used surgical method to treat patients with severe ptosis, which can reduce postoperative infection and recurrence. Clinical studies have shown that patients experience anxiety before any operation is planned, even for minimally invasive surgery, and the prevalence of anxiety could be as high as $50-70 \%$ (6). The eye is one of the most delicate and most important organs of the human body, and the perioperative period has a more profound impact on the psychological emotions of patients (7). This study employed psychological intervention to provide perioperative auxiliary care for patients with severe blepharoptosis treated with autologous fascia lata frontalis suspension to improve their anxiety and depression, thereby promoting the smooth progress of the operation and improving the quality of the surgery. The results are reported as follows. We present the following article in accordance with the CONSORT reporting checklist (available at http://dx.doi.org/10.21037/ apm-21-345).

\section{Methods}

\section{Research subjects}

Ninety-two patients with severe blepharoptosis who underwent autologous fascia lata frontal muscle suspension in our hospital from February 2018 to January 2020 were selected as the research subjects. The inclusion criteria were as follows: (I) patients meeting the diagnostic criteria for severe ptosis: the upper eyelid was lower than the normal position by more than $5 \mathrm{~mm}$; (II) patients meeting the indications for autologous fascia lata frontalis suspension; and (III) patients with normal cognitive function and understanding of the pros, cons, and risks of the operation, as well as the content of the study plan. Included patients signed an informed consent form and were willing to cooperate with the evaluation of various research indicators and follow-up work. The exclusion criteria were as follows: (I) cases complicated with serious diseases, and those unsuitable for surgery; (II) patients with a previous history of mental illness; (III) patients with other eye diseases; and (IV) patients with incomplete data collection required for the research protocol. The aforementioned patients were numbered according to the order of surgery, and were divided into a control group (46 cases) and an observation group (46 cases) using a numerical random table method. All procedures in this study involving human participants were performed in accordance with the Declaration of Helsinki (as revised in 2013). The study protocol was approved by the ethics committee of Second Affiliated Hospital Zhejiang University College of Medicine.

\section{Study methods}

Both groups of patients received routine nursing intervention during the perioperative period, and the observation group also received psychological intervention during this period.

\section{Autologous fascia lata frontal muscle suspension surgery}

Both groups of patients were operated on by the same medical staff. The operation was performed according to the following sequence: local anesthesia, skin incision, autologous fascia lata, fixation of fascia lata to the tarsal plate, frontal muscle, double eyelid formation, and suture of the incision. 


\section{Routine perioperative care of patients}

(I) Preoperative care: routine preoperative visits were conducted for all patients who decided to undergo surgery. The surgical plan, operation time, surgical staff, preoperative preparation arrangements, perioperative patient cooperation matters, precautions, etc. were explained to patients, and their questions were clearly and carefully answered. Antibiotic eye drops were given 3 days before surgery, and patients were assisted in completing various preoperative laboratory and eye index tests. The patients' vital signs were also checked.

(II) Intraoperative care: after entering the room, the patients' vital signs were routinely monitored, their emotional state was understood, and appropriate comfort was provided. Also, the importance of intraoperative cooperation with medical staff, such as the method of opening eyes during the operation, was reiterated.

(III) Post-operative care: ice was applied to the surgical dressing site 30 minutes after surgery, and the surgical incision was disinfected every day. The patients' postoperative pain feeling was understood, and those with intolerable pain were given medication for pain relief. The doctor's advice to care for the lower eyelid traction line of patients was implemented, as well as cleaning and disinfection during the process to prevent infection. After the operation, the patients were given systemic antibiotics and antibiotic eye drops to prevent infection. Three days after the operation, the patients were instructed to exercise with closed eyes, mainly via blinking movements, eye position fixation, eye movement, frontal muscle flap, and orbicularis oculi muscle massage to prevent swelling around the eyelids.

(IV) Discharge guidance: the notice of discharge precautions was distributed and read to the patients individually. The patients' questions about discharge precautions were patiently answered, emphasizing eyelid protection-related measures, such as avoiding spicy and hard foods, coughing when eating, and preventing irritation and stretching of wounds. The time and items of follow-up return to the hospital were also clarified.

\section{Perioperative psychological intervention}

After the patients decided to undergo surgery, they were assigned to nurses trained in psychological nursing- related professional knowledge to conduct pre-operative psychological interventions. This involved the following: (I) diagnosis of the patient's psychological condition, and communication based on a comprehensive understanding their individual information and conditions. Also, the patient's cognition of the disease, as well as the type and intensity of their psychological response (anxiety, fear, depression, dependence, loneliness, guess and doubt, anger, high expectation, denial, etc.) were assessed in order to identify the cause of the patient's psychological response, and a targeted psychology nursing program plan was formulated. (II) With a sincere attitude and a communication method suitable for the patient, the relevant disease knowledge and condition were explained to the patients, and the treatment and prognosis of the previous similar cases conducted in the hospital were introduced, as were the technical team, hardware facilities, management level, etc. Patients were provided with a correct understanding of the discomfort that may be caused during the operation, such as pain, and were at the same time made aware that excessive anxiety and depression during the perioperative period will negatively impact the results of the operation. Through this, we ensured that patients would fully understand the entire treatment and nursing process, and have confidence in the treatment and care. This would relieve their anxiety, fear, depression, and other emotions caused by their worries about the treatment effect and prognosis, and encourage them to actively cooperate and strictly abide by the medical staff's various treatments and medical advice. (III) We also aimed to create a good surgical environment and atmosphere, and played soothing background music as during patient's surgery and postoperative hospitalization to relieve or transfer the patient's tension and fear to some extent. (IV) Before the operation, the patient's tension was evaluated. If the patient's nervousness and fear were serious, they were guided to practice mindfulness meditation, deep breathing, empathy, and other methods to relieve their anxieties. (V) For patients who were nervous and fearful during the operation, the surgical procedure was promptly explained to them so that they realize that the operation is not scary and are able to relax. (VI) The relevant information about the surgical treatment was explained to the patient after the operation, so as to bolster their confidence in the postoperative recovery process. Clarifying the postoperative care measures and precautions using language suitable for the patient can help them to remain relaxed. Close attention was paid to the postoperative pain of the patient. Patients with 
mild pain were relieved by promoting a positive recovery attitude, explaining the cause and duration of the pain, and diverting attention. However, for patients with intolerable pain, medication was used to reduce the pain and its influence on their emotional state. (VII) Upon meeting the discharge indications, a discharge psychological assessment was conducted, which involved patiently listening to the patient's narration, summarizing the patient's psychological problems, and providing suitable guidance. Reliable contact information was also provided, and patients were informed that any abnormal situation could be communicated with medical staff, in order to reduce the anxiety and depression regarding problems that may occur after discharge.

\section{Observation indicators}

The two groups of patients were evaluated using the HAM-A and HAM-D scales when deciding on surgery, as well as before and after surgery, and the results were compared between the groups. The numerical rating scale (NRS) for surgical site pain of was evaluated at $1 \mathrm{~h}$ and $6 \mathrm{~h}$ postoperatively, and the results were compared between the groups. The intra- and post-operative complications of patients were collected, sorted, and compared between the two groups. The medical staff who performed the operations comprehensively evaluated the compliance rate of the two groups of patients during the perioperative period and compared the results. One day before discharge, the two groups of patients were surveyed regarding nursing satisfaction, and the results were compared.

\section{HAM-D and HAM-A evaluation}

For patients who decided to operate, two trained physicians conducted HAM-A and HAM-D evaluations at 1 hour before the operation, and 3 days after the operation. The two assessment physicians talked to the respondent together and observed their emotional condition over the preceding week. They also ensured that patients understood the assessment one-by-one according to the items in the assessment form, and each were scored independently. All items used a 5-level scoring method ranging from 0 to 4 points. The standards for each level were: 0 , asymptomatic; 1, mild; 2 , moderate; 3 , severe; and 4 , extremely severe. Following the evaluation, if the total score difference between the two doctors did not exceed $5 \%$, the average of the two evaluation results was used as the reported value. The highest total score of HAM-A is 29 points, with $>14$ points signifying anxiety; while the highest total score for HAM-D is 24 points, with $>17$ points representing depression.

\section{NRS assessment}

All patients were evaluated for headache at the surgical site by trained nurses at $6 \mathrm{~h}$ and $24 \mathrm{~h}$ postoperatively. Before the evaluation, a scale with $0-10 \mathrm{~cm}$ was used to explain the relationship between pain perception and the scale to patients, with 0 points representing painlessness and 10 points signifying severe pain. The patient then selected the appropriate corresponding score on the scale according to their pain perception during the evaluation, which was considered the NRS score of patients.

\section{Evaluation of compliance rate}

The patient's attending physician and responsible nurse evaluated their compliance rate during the perioperative period. Four items, including patient's perioperative preparation, intraoperative cooperation, postoperative diet, and postoperative eye-closing exercises, were scored. The total score of each item was 25 points (total score of 100 for the four items). Scores over 90 were considered full compliance, scores 60-89 were representative of partial compliance, and scores $<60$ signified non-compliance. The compliance rate was calculated according to following formula: compliance rate $=$ full compliance rate + partial compliance rate.

\section{Observation of complications}

Inquiries were made into the patient's feelings during and after the operation, and the condition of the patient's operation site was observed. The following conditions were regarded as complications: bleeding, local edema, granuloma, irregular eyelid contour, frontal cellulitis, and eyelid asymmetry.

\section{Nursing satisfaction rate survey}

All patients were surveyed regarding their nursing satisfaction rate 1 day before discharge. Patients indicated their satisfaction with the perioperative nursing attitude, nursing professionalism, the communication of nursing staff, the comfort of the nursing operation, etc. by rating their experience as either significantly satisfied, satisfied, or dissatisfied. The satisfaction was calculated according to the following formula: satisfaction rate $=$ (significantly satisfied + satisfied)/total number of people surveyed $\times 100 \%$. 
Table 1 Comparison of perioperative anxiety and depression scores between the two groups of patients $\left(x_{ \pm} \pm \mathrm{s}\right)$

\begin{tabular}{|c|c|c|c|c|c|c|c|c|}
\hline Group & \multicolumn{4}{|c|}{ HAM-A score (points) } & \multicolumn{4}{|c|}{ HAM-D score (points) } \\
\hline $\begin{array}{l}\text { Control group } \\
(n=46)\end{array}$ & $15.03 \pm 2.04$ & $15.67 \pm 3.21$ & -1.141 & 0.257 & $17.82 \pm 2.24$ & $17.27 \pm 2.34$ & 1.220 & 0.225 \\
\hline $\begin{array}{l}\text { Observation group } \\
(\mathrm{n}=46)\end{array}$ & $14.24 \pm 1.58$ & $13.02 \pm 1.17$ & 4.209 & $<0.001$ & $16.81 \pm 1.83$ & $16.32 \pm 2.07$ & 1.203 & 0.232 \\
\hline$P$ value & 0.041 & $<0.001$ & & & 0.020 & 0.031 & & \\
\hline
\end{tabular}

$\bar{x} \pm \mathrm{S}$, mean \pm standard deviation; $\mathrm{n}$, the number of cases; $\mathrm{T}$ value, the statistical value of the $t$-test; $\mathrm{P}$ value, the probability of occurrence; HAM-A, Hamilton Anxiety; HAM-D, Hamilton Depression.

Table 2 Comparison of NRS scores between two groups of patients at different stages after surgery $\left(\bar{x}_{ \pm}\right)$

\begin{tabular}{lccc}
\hline \multirow{2}{*}{ Group } & \multicolumn{2}{c}{ Time (points) } & T value \\
\cline { 2 - 3 } & Postoperative 6 $\mathrm{h}$ & Postoperative 24 $\mathrm{h}$ & \\
\hline Control group $(\mathrm{n}=46)$ & $3.02 \pm 0.28$ & $1.69 \pm 0.11$ & 29.985 \\
Observation group $(\mathrm{n}=46)$ & $2.56 \pm 0.16$ & $1.23 \pm 0.04$ & 54.695 \\
T value & 9.674 & 26.654 & $<0.001$ \\
$P$ value & $<0.001$ & $<0.001$ & \\
\hline
\end{tabular}

$\bar{x} \pm \mathrm{S}$, mean \pm standard deviation; $\mathrm{n}$, the number of cases; $\mathrm{T}$ value, the statistical value of the $t$-test; $\mathrm{P}$ value, the probability of occurrence; NRS, numerical rating scale.

\section{Statistical analysis}

SPSS19.0 software package was used for the statistical analysis. Measurement data was represented by $\bar{x} \pm \mathrm{s}$, and was analyzed using the $t$-test. Count data was analyzed by $\chi^{2}$, and rank count data was subjected to the rank-sum test. $\mathrm{P}<0.05$ was considered to indicate a statistically significant difference.

\section{Results}

\section{Comparison of basic data between the two groups}

The control group included a total of 61 cases of severe eyelid ptosis, 21 males with 28 eyes, 25 females with 33 eyes, with an average age of $38.35 \pm 7.07$ years, 21 cases of congenital ptosis, 21 cases of traumatic ptosis, and four cases of palpebral fissure. At the time of surgery, the Hamilton Anxiety (HAM-A) and Depression (HAM-D) rating scores were determined to be $14.04 \pm 1.57$ and $16.81 \pm 2.42$, respectively. In the observation group, there were 62 cases of severe eyelid ptosis, including 20 males with 28 eyes, 26 females with 34 eyes, with an average age of $38.16 \pm 7.12$ years, 20 cases of congenital ptosis, 23 cases of traumatic ptosis, and three cases of blepharoptosis. The HAM-A and HAM-D scores were $14.12 \pm 1.53$ and $16.92 \pm 2.45$, respectively, at the time of surgery. There were no significant differences in the general information between the two groups of patients $(P>0.05)$.

\section{Comparison of perioperative anxiety and depression scores between the two groups}

The preoperative and postoperative HAM-A and HAM-D scores of the observation group were significantly lower than those of the control group $(\mathrm{P}<0.05)$, as indicated in Table 1.

\section{Comparison of NRS scores between the two groups of patients at different stages after surgery}

The NRS scores of patients in the observation group were markedly lower than those of the control group at $6 \mathrm{~h}$ and $24 \mathrm{~h}$ after surgery $(\mathrm{P}<0.05)$, as shown in Table 2. 
Table 3 Comparison of intra- and post-operative complication rates between the two groups

\begin{tabular}{|c|c|c|c|c|}
\hline Complications & Control group ( $\mathrm{n}=46), \mathrm{n}(\%)$ & Observation group $(n=46), n(\%)$ & $\chi^{2}$ value & $P$ value \\
\hline Bleeding & $1(2.17)$ & 0 & & \\
\hline Eyelid asymmetry & $1(2.17)$ & $1(2.17)$ & & \\
\hline Frontal cellulitis & $1(2.17)$ & 0 & & \\
\hline \multicolumn{5}{|l|}{ Post-operative } \\
\hline Local edema & $2(4.35)$ & $1(2.17)$ & & \\
\hline Granuloma & $1(2.17)$ & $1(2.17)$ & & \\
\hline Complication & $7(15.22)$ & $4(8.70)$ & 3.514 & 0.002 \\
\hline
\end{tabular}

$\mathrm{n}$, the number of cases; $\chi^{2}$ value, the statistical value of chi-square test; $P$ value, the probability of occurrence.

Table 4 Comparison of perioperative compliance rates between the two groups of patients

\begin{tabular}{|c|c|c|c|c|}
\hline Compliance & Control group $(n=46), n(\%)$ & Observation group $(n=46), n(\%)$ & $Z$ value & $P$ value \\
\hline Partial compliance & $17(36.96)$ & $13(28.26)$ & & \\
\hline Non-compliance & $6(13.04)$ & $2(4.35)$ & & \\
\hline Compliance rate & $40(86.96)$ & 44 (95.65) & & \\
\hline
\end{tabular}

$\mathrm{n}$, the number of cases; $Z$ value, the statistical value of rank sum test; $P$ value, the probability of occurrence.

\section{Comparison of intra- and post-operative complication rates between the two groups}

The intra- and post-operative complication rates of the observation group were lower than those of the control group $(\mathrm{P}<0.05)$, as shown in Table 3.

\section{Comparison of perioperative compliance rates between the two groups of patients}

The compliance rate of patients in the observation group was significantly higher than that of patients in the control group $(\mathrm{P}<0.05)$, as indicated in Table 4 .

\section{Comparison of the satisfaction rates of the two groups of patients with care}

The satisfaction rate with perioperative care in the observation group was significantly higher than that of the control group $(\mathrm{P}<0.05)$, as described in Table 5 .

\section{Discussion}

Patients with severe ptosis suffer impaired visual function and a significant change to their facial structure, which has a notably impact on their body and mind. Autologous fascia lata frontal muscle suspension is currently the most widely used program for the treatment of severe blepharoptosis, and is also the most common type of clinical cosmetic surgery (8). Autogenous fascia frontal muscle suspension has obvious advantages over other treatment options and can achieve better clinical efficacy and prognosis. In addition, a nursing plan suitable for patients plays an important role in ensuring the quality of surgery, postoperative recovery, and a positive patient experience with medical treatment $(9,10)$. With the increasing amount of clinical research on the occurrence, development, and treatment of diseases, the influence of psychological and emotional factors in the occurrence and development of diseases, as well as the prognosis of treatment, has received increasing clinical attention (11). Perioperative anxiety and depression have 
Table 5 Comparison of nursing satisfaction rate between the two groups

\begin{tabular}{|c|c|c|c|c|}
\hline Satisfaction & Control group $(n=46), n(\%)$ & Observation group $(n=46), n(\%)$ & $Z$ value & $P$ value \\
\hline Satisfied & $21(45.65)$ & $11(23.91)$ & & \\
\hline Not satisfied & $4(8.70)$ & $1(2.17)$ & & \\
\hline Satisfaction rate & $42(92.30)$ & $45(97.83)$ & & \\
\hline
\end{tabular}

$\mathrm{n}$, the number of cases; $Z$ value, the statistical value of rank sum test; $P$ value, the probability of occurrence.

a significant impact on the stress response level, pain perception, compliance rate, postoperative recovery, and medical experience of patients. Patients with increased pain and emergency response levels, which occur as a result of psychological and emotional factors, will experience further anxiety and depression. This will lead to a reduced rate of compliance and postoperative rehabilitation. Improving the anxiety and depression of surgical patients during the perioperative period is an important aspect for all surgeries. For patients with severe blepharoptosis undergoing autologous fascia lata frontal muscle suspension, due to the particularity and importance of the surgical site, anxiety and depression during the perioperative period is more serious. This study employed psychological intervention based on routine perioperative care to reduce the anxiety and depression of patients during this period, encourage enthusiasm for treatment, promote the effects of surgical treatment, and improve prognosis.

The results showed that patients who also received perioperative psychological intervention had a significant decrease in HAM-A and HAM-D scores 1 day before and after surgery, as well as a decrease in NRS scores at $6 \mathrm{~h}$ and $24 \mathrm{~h}$ after surgery compared with patients who received conventional care alone. Moreover, the perioperative complication rate of patients who received perioperative psychological intervention was lower, and the compliance and satisfaction rates of patients with nursing care increased significantly $(\mathrm{P}<0.05)$. It is suggested that perioperative psychological intervention for severe upper eyelid patients undergoing autologous fascia lata frontalis suspension surgery can effectively improve the patient's negative emotions, thereby improving the overall quality of surgery and the patient's experience with medical treatment, which demonstrates the high clinical of perioperative psychological intervention.

Psychological intervention has been shown to improve the anxiety and depression of patients in many diseases.
At present, psychological intervention includes numerous modalities. For surgical patients, the following three points should be emphasized: (I) providing patients with the necessary information for surgical treatment, ensuring that they correctly recognize their disease, treatment plan, and possible prognosis. (II) Medical staff should pay attention to their language and attitude as well as the patient's understanding of nursing at all times during the process, and implement suitable measures to ensure that the patient understands and is able to make informed decisions. (III) Providing sufficient social support to strengthen the confidence of patients in their disease and the surrounding environment. (IV) Providing a comfortable environment for surgery and hospitalization. Hepp et al. (11) utilized musical intervention for women undergoing cesarean section, which effectively reduced the anxiety and stress of those women. Biglioli et al. (12) implemented psychological interventions for patients who had undergone cross-facial nerve transplantation and platysma transplantation to repair paralyzed eyelids, which effectively improved the patients' negative emotions. Senra et al. (13) adopted psychosocial intervention to treat anxiety and depression in patients with age-related macular degeneration. Tomaszek et al. (14) used preoperative comprehensive confidence support to improve the anxiety and pain of adolescent patients undergoing chest surgery and enhance patient satisfaction with analgesia. White et al. (15) implemented psychosocial treatment for adolescents and adults with autism to effectively reduce their anxiety and depression symptoms. These benign effects of psychological intervention on disease patients indicate that psychological intervention will become an important auxiliary method for future disease treatment and is a developing trend in the clinical treatment of disease.

In summary, psychological intervention can effectively alleviate the negative emotions of severe blepharoptosis patients undergoing autologous fascia lata frontalis suspension, improve their compliance with medical care, 
reduce pain, and increase patient satisfaction, indicating its high clinical value.

\section{Acknowledgments}

Funding: None.

\section{Footnote}

Reporting Checklist: The authors have completed the CONSORT reporting checklist. Available at http://dx.doi. org/10.21037/apm-21-345

Data Sharing Statement: Available at http://dx.doi. org/10.21037/apm-21-345

Conflicts of Interest: Both authors have completed the ICMJE uniform disclosure form (available at http://dx.doi. org/10.21037/apm-21-345). The authors have no conflicts of interest to declare.

Ethical Statement: The authors are accountable for all aspects of the work in ensuring that questions related to the accuracy or integrity of any part of the work are appropriately investigated and resolved. All procedures in this study involving human participants were performed in accordance with the Declaration of Helsinki (as revised in 2013). The study protocol was approved by the ethics committee of Second Affiliated Hospital Zhejiang University College of Medicine (No. 2016031). Included patients signed an informed consent form.

Open Access Statement: This is an Open Access article distributed in accordance with the Creative Commons Attribution-NonCommercial-NoDerivs 4.0 International License (CC BY-NC-ND 4.0), which permits the noncommercial replication and distribution of the article with the strict proviso that no changes or edits are made and the original work is properly cited (including links to both the formal publication through the relevant DOI and the license). See: https://creativecommons.org/licenses/by-nc-nd/4.0/.

\section{References}

1. Shimizu Y, Nagasao T, Shido H, et al. Intra-eyebrow frontalis suspension using inverted Y-shaped short autogenous fascia lata for blepharoptosis with poor levator function. J Plast Reconstr Aesthet Surg 2015;68:49-55.

2. Finsterer J. Ptosis: causes, presentation, and management. Aesthetic Plast Surg 2003;27:193-204.

3. Gundeslioglu AO, Selimoglu MN, Bekerecioglu M. An frontalis sling operation using an autogenous en-bloc, fanshaped tensor fascia lata graft for blepharoptosis. J Plast Reconstr Aesthet Surg 2013;66:224-30.

4. Théra JP, Tiama JM, Konipo A, et al. Treatment of congenital ptosis in a low-income country: polypropylene frontalis sling at the African Institute of Tropical Ophthalmology. J Fr Ophtalmol 2020;43:123-7.

5. Morax S, Benia L. Suspension of the eyelid to the frontal muscle in the surgery of ptosis. Technic and indications. J Fr Ophtalmol 1986;9:461-70.

6. Shepardson RL, Buchholz LJ, Weisberg RB, et al. Psychological interventions for anxiety in adult primary care patients: A review and recommendations for future research. J Anxiety Disord 2018;54:71-86.

7. Bouazza M, Elbelhadji $M$, Mchachi A, et al. Treatment of congenital ptosis by frontalis suspension with monofilament polypropylene suture: results of a study of 21 cases. J Fr Ophtalmol 2014;37:520-5.

8. Silvério J, Sugano DM, Lucci LM, et al. Frontalis suspension with polytetrafluorethylene for the treatment of blepharoptosis. Arq Bras Oftalmol 2009;72:79-83.

9. Junceda-Moreno J, Suárez-Suárez E, Dos-SantosBernardo V. Treatment of palpebral ptosis with frontal suspension: a comparative study of different materials. Arch Soc Esp Oftalmol 2005;80:457-61.

10. Ruban JM, Mallem M, Tabone E, et al. A new biomaterial in surgery of ptosis with frontalis suspension: wide pore PTFE. J Fr Ophtalmol 1995;18:207-19.

11. Hepp P, Hagenbeck C, Gilles J, et al. Effects of music intervention during caesarean delivery on anxiety and stress of the mother a controlled, randomised study. BMC Pregnancy Childbirth 2018;18:435.

12. Biglioli F, Zago M, Allevi F, et al. Reanimation of the paralyzed lids by cross-face nerve graft and platysma transfer. J Craniomaxillofac Surg 2018;46:521-6.

13. Senra H, Macedo AF, Nunes N, et al. Psychological and Psychosocial Interventions for Depression and Anxiety in Patients With Age-Related Macular Degeneration: A Systematic Review. Am J Geriatr Psychiatry 2019;27:755-73.

14. Tomaszek L, Cepuch G, Fenikowski D. Influence of preoperative information support on anxiety, pain and satisfaction with postoperative analgesia in children and adolescents after thoracic surgery: A randomized double 
blind study. Biomed Pap Med Fac Univ Palacky Olomouc Czech Repub 2019;163:172-8.

15. White SW, Simmons GL, Gotham KO, et al. Psychosocial Treatments Targeting Anxiety and Depression in Adolescents and Adults on the Autism Spectrum: Review

Cite this article as: Cai $\mathrm{H}, \mathrm{Xu} \mathrm{S}$. A randomized trial of psychological intervention on perioperative anxiety and depression of patients with severe blepharoptosis undergoing autologous fascia lata frontal muscle suspension. Ann Palliat Med 2021;10(3):3185-3193. doi: 10.21037/apm-21-345 of the Latest Research and Recommended Future Directions. Curr Psychiatry Rep 2018;20:82.

(English Language Editor: A. Kassem) 